\title{
OH Masers Associated with IRAS Point Sources
}

\author{
M. R. W. Masheder \\ Department of Physics, University of Bristol, Tyndall Avenue, Bristol, \\ BS8 1TL, UK (email : mike.masheder@bristol.ac.uk)
}

R. J. Cohen

Jodrell Bank Observatory, Macclesfield, Cheshire, SK11 9DL, UK

\author{
N. L. Martin-Hernandez \\ Kapteyn Institute, Postbus 800, 9700 NV Groningen, Netherlands
}

\begin{abstract}
We report a search for masers from the $\Lambda$-doublet of the ground-state of $\mathrm{OH}$ at $18 \mathrm{~cm}$, carried out with the Jodrell Bank Lovell Telescope and with the $25 \mathrm{~m}$ Dwingeloo telescope. All objects north of $\delta=-20^{\circ}$ which appear in the IRAS Point Source Catalog with fluxes $>1000 \mathrm{Jy}$ at $60 \mu \mathrm{m}$ and $100 \mu \mathrm{m}$ were observed in all four lines and both circular polarisations over the required velocity range. Preliminary results of 52 maser sources are reported here.
\end{abstract}

\section{Introduction}

Masers in the transitions within the $\Lambda$-doublet of the ${ }^{2} \Pi_{3 / 2}, \mathrm{~J}=3 / 2$ ground state of $\mathrm{OH}$ are often found to be associated with regions of recent massive star formation and ultra-compact HII objects are often found within such regions. These may often be recognised by their far infrared (FIR) properties. To investigate the relationship between $\mathrm{OH}$ masers and UCHII's we have searched all objects with $\delta>-20^{\circ}$ and with fluxes at 60 and $100 \mu \mathrm{m}>1000 \mathrm{Jy}$ in the IRAS Point Source Catalog. These simple flux-limit criteria allow us to investigate the FIR colours of the maser objects found, along the lines of Wood and Churchwell (1989). There are 188 objects matching the criteria, but because of clustering it was not possible to observe them all separately. This work is intended to complete that started by Cohen et al. (1988) by improving the sensitivity in the main lines by a factor of $\sim 4$ and adding observations of the satellite lines.

\section{Observations}

Most of the observations were carried out in the summer of 1993 using the Lovell Telescope at Jodrell Bank, but radio interference prevented the completion of the work. Further observations were attempted in 1994, but again the survey was not completed because of interference. The Dwingeloo $25 \mathrm{~m}$ telescope was available to us in 1998 and was used to finish the search and check previous 
possible detections. The receivers at the two observatories were similar. Each had a dual front-end giving the two hands of circular polarisation with a system noise of about 35-40K. At each observatory a dual 512-channel auto-correlator spectrometer was used with a bandwidth of $625 \mathrm{kHz}$. After Hanning smoothing, the velocity resolution is about $0.45 \mathrm{~km} / \mathrm{s}$.

The survey is complete in that every target source was observed at least once in all four lines and both polarisations. The sensitivity to maser emission is not uniform because of complications due to observing multiple objects in a telescope beam. The average $3 \sigma$ detection limit is $0.26 \mathrm{Jy}$ with almost all values within a factor 2 of this. Some previously well-known masers were observed at only their known velocity so it is possible that emission more than $50 \mathrm{~km} / \mathrm{s}$ away could have been missed. Only in this sense is the survey not complete.

\section{Preliminary Results}

$\mathrm{OH}$ masers were found associated with 52 of the IRAS Point Source objects searched. Where possible, these associations were based on measurements of maser positions in the literature. For most objects with Galactic longitudes $<49^{\circ}$, the positions are due to Caswell (1998 and Priv. Comm.). For most of the others, measurements due to Argon et al. (2000) were used. These results are listed in a table available on request by email to the first author. Not counted in this list are :

- $\mathrm{OH} / \mathrm{IR}$ stars observed at $1612 \mathrm{MHz}$

- Many instances of quasi-thermal emission and absorption. In some cases this is a matter of subjective judgement, based on the symmetry between the satellite line shapes and the absorption in the main lines which usually accompanies this emission. Weak masers may have been missed in these cases.

- Well known masers whose positions are not close to the IRAS positions, for example the bright masers in the W51 region.

\section{Discussion}

\subsection{Comparison between $\mathrm{OH}$ and far-infrared emission}

$\mathrm{OH}$ masers have for many years been associated with star-forming regions and on a smaller scale with the ultra-compact HII regions (UCHII) that surround newly formed hot stars (eg Zuckerman et al., 1965; Cook, 1966; Reid et al., 1980; Gaume and Mutel, 1987). Such objects are bright in the far infra-red, so many are expected in our flux-limited sample. Because the targets were selected on the basis of flux only, it is important to see if the detected objects display properties associated with UCHIIs. This is shown in Figure 1, an IRAS 2-colour plot along the lines given by Wood and Churchwell (1989) [WC]. It is clear that all but one of our masers (filled circles) fall in the box (shown) in which WC expect to find UCHII's. This is clear evidence for the expected tight correlation between $\mathrm{OH}$ masers and UCHII's.

As the pumping of $\mathrm{OH}$ masers is believed to involve infra-red radiation, it may be interesting to compare the strengths of $\mathrm{OH}$ and FIR emission. This is shown in Figure 2 in which the $\mathrm{OH}$ maser luminosity is plotted vs. the FIR 


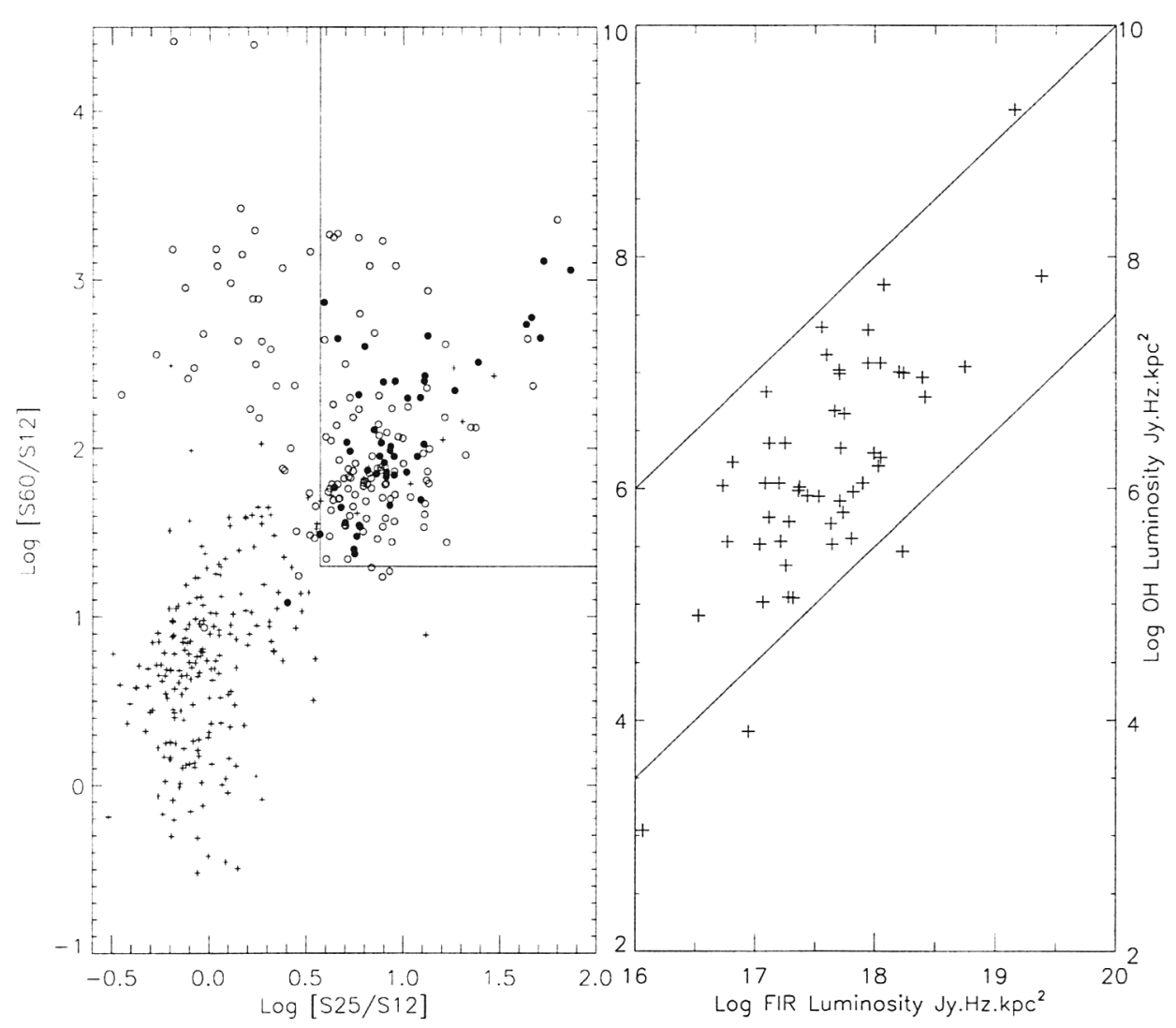

Figure 1. (left) Two-colour plot of IRAS point sources (after WC). + show WC's random control sample, all the entries in a box $2^{\circ}$ square in the Galactic plane at longitude $40^{\circ}$.

o show our target sample, S60 and S100 > $1000 \mathrm{Jy}$

- show those objects found to be associated with $\mathrm{OH}$ masers in the present study. All but one have FIR colours in the ranges expected for ultra-compact HII regions, as shown by the box as top right.

Figure 2. (right) $\mathrm{OH}$ maser luminosity plotted versus FIR luminosity. The diagonal guidelines are for $\mathrm{OH}$ proportional to FIR 
luminosity between 8 and $135 \mu \mathrm{m}$ of the associated IRAS source calculated by a method due to Emerson (1988). The diagonal lines are for simple proportionality and are intended only to guide the eye. For the purposes of this plot, kinematic distances were used which were derived from a simple flat Galactic rotation curve using a constant velocity of $220 \mathrm{~km} . \mathrm{s}^{-1}$ and a Galactocentric distance of $8.5 \mathrm{kpc}$ for the Sun. For most of the objects for which there is a distance ambiguity, the near distance was adopted. Poor values of distance found in this over-simple method merely move points parallel to the guide lines. The $\mathrm{OH}$ luminosity appears to be proportional to that at FIR, but the correlation is not tight - the lines shown are a factor of 300 apart. The apparent proportionality may be a selection effect rather than anything to do with the infra-red pumping mechanisms.

\subsection{Other points}

Of the 52 IRAS sources found to be associated with $\mathrm{OH}$ masers, only 12 were detected in the satellite lines. 10 were detected at $1720 \mathrm{MHz}$, of which 6 were not detected at $1612 \mathrm{MHz}$ and 6 sources were detected at $1612 \mathrm{MHz}$, of which 2 were not detected at $1720 \mathrm{MHz}$. One object, 20255+3712, was detected in the $1720 \mathrm{MHz}$ line and no other line.

It was noticed that quasi-thermal absorption and emission from more diffuse $\mathrm{OH}$ were commonly observed. This has prompted Masheder and Gray to carry out a more detailed analysis. (See a poster at this conference and a later paper)

\section{The future}

It is planned to publish these results in full later in 2001. In addition to a printed paper, both the individual spectra and the derived tables will be made available on-line at the CDS in Strasbourg via the Vizier facility.

Acknowledgments. The authors thank NFRA, Dwingeloo for their hospitality and for the use of the $25 \mathrm{~m}$ telescope. Thanks are also due to Gideon Hallett for his invaluable help in taking the observations at Jodrell Bank and in their subsequent processing.

\section{References}

Argon A. L., Reid M. J. \& Menten K. M. 2000 ApJS, 1291

Caswell J. L. private communication

Caswell J. L., 1998 MNRAS, 297215

Cohen R. J., Baart E. E. \& Jonas J. L. 1988 MNRAS, 231205

Cook A. H. 1966 Nature, 211, 502

Gaume R. A. \& Mutel R. L. 1987 ApJS65 193

Emerson J. 1988 In 'Formation and evolution of low mass stars' (Reidel) p193

Reid M. J., Haschick A. D., Burke B. F., Moran J. M., Johnston K. J., \& Swenson G.W. 1980 ApJ, 23989

Wood and Churchwell 1989 ApJ, 340265 [WC]

Zukerman B. and Palmer P. 1974 ARA\&A, 12279 